\title{
Evaluation of pain relief treatment and timelines in emergency care in six European countries and Australia
}

\author{
Andrew D Xia $\mathbb{D}^{\prime}$ \\ Sara L Dickerson (ID ${ }^{2}$ \\ Andrew Watson ${ }^{3}$ \\ Mika Nokela (D) ${ }^{4}$ \\ Sam Colman (iD ${ }^{5}$ \\ Agota Szende ${ }^{6}{ }^{6}$
}

'Mundipharma International Limited, Cambridge, UK; ${ }^{2}$ Mundibiopharma Limited, Cambridge, UK; ${ }^{3}$ Canterbury and Concord Hospitals, Sydney, NSW, Australia; ${ }^{4}$ Covance Market Access Inc., Solna, Sweden; ${ }^{5}$ Covance Market Access Inc, Sydney, NSW, Australia; ${ }^{6}$ Covance Market Access Inc., Leeds, UK
Correspondence: Sara L Dickerson Mundibiopharma Limited, Cambridge Science Park, Milton Road, Cambridge CB4 OAB, UK

$\mathrm{Tel}+4401223397684$

Email sara.dickerson@mundipharma.com
This article was published in the following Dove Press journal: Open Access Emergency Medicine

Purpose: Inadequate relief of pain is common in prehospital and hospital emergency department (ED) settings. We investigated pain treatments and timelines in patients receiving pre-hospital and hospital ED care to provide insight into potential approaches to reduce the burden of trauma-related pain.

Patients and methods: In this observational, retrospective chart review, patients had received emergency care for musculoskeletal trauma injuries and analgesic treatment for moderate-to-severe pain in Belgium, France, Germany, Italy, Spain or Sweden. As inhaled low-dose methoxyflurane (LDM) is used extensively in Australia but was not widely available in Europe at the time of this analysis, data from Australia were collated to provide insight into the potential utility of this analgesic in Europe. The primary endpoint was time to administration of first pain relief treatment following arrival of paramedic/ED care.

Results: Randomly selected physicians $(n=189)$ collated data from 856 patients (Europe: $\mathrm{n}=585$; Australia: $\mathrm{n}=271$ ) via an online survey. Time to first pain relief treatment varied between countries and was significantly longer across Europe versus Australia (mean [SD] 38.1 [34.7] vs 29.9 [35.5] mins; $P=0.0017$ ). Patients from Australia who received LDM experience a shorter mean (SD) time to first pain treatment following arrival of emergency care versus patients who received other analgesics (propensity score matched $[n=85]$ per group: 21.7 [24.2] vs 39.1 [43.0] mins; $P=0.0013$ ). Across all countries, mean (SD) time to first analgesic was shorter when treatment was administered by paramedics versus hospital ED staff (15.7 [14.7] vs 49.1 [38.4] mins).

Conclusions: While there was a large variation in analgesia timelines across countries, mean times are shorter in Australia compared with Europe overall. In Australia, use of LDM was associated with a significantly shorter time from emergency assistance to first pain treatment compared with non-LDM treatments. Further studies are needed to investigate the utility of LDM in Europe.

Keywords: methoxyflurane, pain management, ambulance, retrospective study, emergency medical service, analgesia

\section{Plain language summary}

While timely analgesia is important to avoid unnecessary suffering in trauma patients, oligoanalgesia is commonly reported in this setting. This observational, retrospective chart review investigated time to analgesic treatment following arrival of paramedic/emergency care in patients with acute musculoskeletal injuries who experience moderate-to-severe pain. The potential impact of inhaled low-dose methoxyflurane (LDM), which was not widely available in Europe at the time this study was conducted, was also investigated using data 
obtained from patients in Australia where LDM is widely used. There was a large variation in time to analgesic treatment across the European countries studied (Belgium, France, Germany, Italy, Spain and Sweden, $n=585$ ). In a cohort of patients from Australia $(n=271)$, use of LDM was associated with a significantly shorter time from arrival of emergency assistance to first pain treatment compared with other pain relief treatments. Utilization of rapidly acting, non-opioid, needle-free analgesics such as LDM by paramedics in Europe has potential to reduce the time to first pain relief treatment in adults with acute musculoskeletal injuries and moderate-to-severe pain.

\section{Introduction}

Effective pain management is a key component of care for the estimated 38 million injured people treated in European hospital emergency departments (ED) every year. ${ }^{1}$ Undertreated pain not only poses an immediate burden to patients, but may also result in psychological consequences, physiological effects which delay healing, and lead to increased healthcare resource utilization. ${ }^{2,3}$ However, inadequate relief of traumarelated pain is common despite recommendations that patients with moderate-to-severe pain receive analgesia with 15-20 mins of arriving at hospital EDs. ${ }^{4,5}$ Studies suggest the majority of patients attending European EDs with moderate-tosevere pain receive no analgesia, and high levels of pain are frequently reported by patients when discharged from EDs. ${ }^{6-9}$ Furthermore, suboptimal management of moderate-to-severe trauma pain is also common in pre-hospital emergency care settings. $^{10,11}$

Patients may face numerous barriers to effective control of trauma-related pain, including inadequate assessment of their pain and prolonged waiting times in hospital EDs. ${ }^{12}$ Analgesics commonly used for moderate-to-severe pain in emergency settings including $\mathrm{N}_{2} \mathrm{O}$, ketamine and opioids are also associated with limitations. ${ }^{12}$ For example, while opioids can provide effective analgesia for selected patients with severe pain, some health care professionals (HCPs) are reluctant to administer these analgesics due to concerns regarding patient drug-seeking behavior or addiction, increased demands for patient monitoring and fear of masking other symptoms of trauma. ${ }^{12,13}$ Delays to analgesia may also result from difficulties obtaining intravenous (IV) access in some emergency settings. Also, the bulky equipment (cylinders and breathing apparatus) required for $\mathrm{N}_{2} \mathrm{O}$ treatment can limit its practicality in some prehospital settings. ${ }^{12}$

Inhaled, low-dose methoxyflurane (LDM) is a nonopioid analgesic. The efficacy and safety of LDM as an analgesic for trauma-associated pain and for medical procedures have recently been reviewed. ${ }^{14}$ Patients can self-administer this volatile fluorinated hydrocarbon under medical supervision via the handheld, Penthrox ${ }^{\circledR}$ "green whistle" inhaler. LDM provides rapid pain relief, occurring within 6-10 inhalations, which is maintained for 25-30 mins (intermittent inhalation may provide longer relief). ${ }^{15}$ LDM has been licensed in Australia for over 30 years where it has been widely used to provide short-term relief of acute pain in adults and children in hospital EDs and prehospital emergency care settings. ${ }^{16,17}$ In Europe, LDM was approved in 2015 for emergency relief of moderate-to-severe trauma-associated pain in conscious adults, based on evidence from the Phase III STOP! study (NCT01420159). This study demonstrated significantly greater reductions in pain scores with LDM compared with placebo and a rapid onset of pain relief (median of 4 mins) in adult and adolescent patients attending UK hospital EDs due to trauma injuries. ${ }^{18}$

Due to its non-opioid mechanism of action, rapid onset of action and portability, LDM has the potential to overcome several barriers associated with the use of some more established analgesic agents in Europe. The aim of this retrospective, real-world study was to obtain greater insight into pain treatments and timelines to pain relief experienced by patients with trauma-related injuries in Europe. Given that LDM was not yet marketed in Europe at the time of this analysis, data from Australia, where LDM is used extensive, were also analyzed to provide insight into the potential impact of this treatment on analgesic on time to administration of first pain relief following arrival of paramedic/ED care.

\section{Methods}

\section{Study design}

This retrospective chart review was conducted to examine timelines and treatment outcomes in medical emergency situations where pain relief was administered to patients in 6 European countries (Belgium, France, Germany, Italy, Spain and Sweden) and Australia. Data were collected via an online survey which was completed by a random sample of physicians working in the ED who were recruited using nationally representative managed databases. Physicians included in this survey had practiced medicine for $>3$ years, worked at their ED during the prior 6 months and had personally managed or treated $>3$ adult patients per week in emergency settings. 
Each physician was asked to identify four eligible patients ( $\geq 18$ years of age) who they had treated most recently within the prior 6 months. Eligible patients had sustained acute musculoskeletal trauma injuries (compression, dislocation, laceration, sprain/strain, contusions, other type of injury of the lower limbs, upper limbs, head, abdominal, chest/thorax, spine, or other site) which required paramedic or other form of emergency care. All patients had moderate-to-severe pain, which had been assessed using a numerical rating scale (NRS; $\geq 4[0-10$ scale]) or visual analog scale (VAS; $\geq 40$ [0-100 scale]) and received analgesic treatment. Patients must have remained conscious throughout the emergency event and were excluded if an altered level of consciousness had occurred due to any cause, including head injury, drugs, or alcohol. Patients were also excluded who self-reported clinically significant renal impairment or had clinical evidence of respiratory depression or cardiovascular instability at the time of the emergency event.

\section{Study objectives and data collection}

The primary objective was to determine the time to administration of the first analgesic following arrival of paramedic or ED HCP assistance. In the patients from Australia, time to pain relief was also compared between individuals receiving LDM versus those receiving other analgesics as their first pain relief treatment (non-LDM). Secondary objectives included assessment of the patients' demographic and clinical characteristics, including the pain experienced; investigating the association between time to analgesic treatment and time to discharge from hospital; and obtaining insight into emergency pain treatment, including the first and subsequent pain medications administered, type of HCP administering analgesia and hospitalization details.

Physicians reviewed the patients' medical charts and reported anonymized patient-level information using an online data abstraction form. Data collected included: characteristics of the patient, trauma/injury and associated pain; time from emergency call to the arrival of a paramedic or emergency HCP; time from arrival of a paramedic or emergency HCP to administration of first pain relief treatment and pain relief treatments received. The following time durations were also recorded: arrival of paramedic to arrival at hospital ED; arrival at hospital ED to ED admission and ED admission to hospital discharge. Time to surgery during hospitalization, time to admission to intensive care unit (ICU) and duration of ICU stay were also recorded (data not reported). The physicians also rated the severity of the injury based on their clinical judgment (mild, moderate or severe). Pain severity was based on NRS or VAS pain scale data recorded in the patient charts. In Australia, where LDM is used in routine practice, physicians were asked to identify patients treated with LDM or other pain relief medications.

\section{Statistical methods}

Data obtained from all physicians and patients meeting the inclusion criteria were included in the analyses. The primary endpoint (time to first analgesic administration following arrival of paramedic or ED HCP) and other time interval endpoints were compared between the European and Australian datasets using 2-sided Student's $t$-tests. T-tests were also used to compare time interval endpoints in the LDM and non-LDM Australian subgroups. Due to the rightskewed nature of the primary endpoint data, a non-parametric treatment group comparison was performed using Wilcoxon Rank-Sum test for sensitivity. Patient demographic and clinical characteristics of the Australian LDM and non-LDM groups were compared using $t$-tests and Chisquare tests, as appropriate. Due to the non-randomized design of this study, propensity score matching (PSM) was used to adjust for confounding between LDM and non-LDM Australian subgroups. The propensity scores for LDM treatment were evaluated using a logistic model with baseline covariates for age, gender, geographic location, race, injury type, injury severity and pain severity, and patients were matched using a nearest neighbor caliper algorithm set at 0.25 standard deviations (SD). No statistical comparisons were conducted between the European countries.

All statistical tests were two-sided and used a 5\% significance level. As the primary analysis was descriptive in nature, a power calculation for comparative analysis was not applicable. A sample of 100 patients per country was expected to provide good precision for the primary endpoint. For the comparisons between LDM and nonLDM Australian subgroups, 100 patients per treatment group provided a maximum of $80 \%$ power to detect a small-to-medium Cohen's d effect size of 0.40 with a significance level of $5 \%$.

\section{Ethical issues}

This research survey was conducted in compliance with the protocol and the Code of Conduct of the European Pharmaceutical Market Research Association (EphMRA) 
and the Australian Market and Social Research Society (AMSRS). All data were collected by physicians, no patient-identifying data (e.g., initials, date of birth or ZIP code) were obtained and patient anonymity was strictly guaranteed. Also, no tabulation/record form linking the patient number and name was used.

\section{Results \\ Physician characteristics}

Between December 2016 and February 2017, the online survey was completed by 189 eligible physicians from 145 hospitals, of whom $n=48$ and $n=141$ were recruited in Australia and Europe (Belgium n=19, France $n=28$, Germany $n=27$, Italy $n=27$, Spain $n=25$, Sweden $n=15$ ), respectively.

Most physicians were ED specialists (57\%) or specialized in internal medicine $(18 \%)$ or general medicine (15\%). The mean (SD) time physicians had been practicing in their medical specialty was similar in Europe (14.4 [6.6] years) and Australia (16.4 [8.1] years). Physicians in Germany (8.8 [3.5] years) and France (19.2 [6.8] years) had been practicing for the shortest and longest durations, respectively. A median (range) of 60 (4-300) patients was managed in the ED by each physician per week. Physicians in Germany (95 [50-150), Spain (90 [18250]), France (90 [20-300]) and Belgium (80 [10-120]) managed more patients per week compared with their counterparts in Australia (40 [5-150]), Italy (30 [4-100]) and Sweden (30 [5-60]).

\section{Patient characteristics}

Medical charts of 856 eligible patients were reviewed, including 585 from Europe (48 to 113 charts per country) and 271 from Australia. Of the Australian patients, $n=128$ were treated with LDM as their first analgesic (LDM group) and $n=118$ received no LDM (non-LDM group). Data from $n=25$ Australian patients were not allocated to LDM/non-LDM groups as their first pain treatment was administered prior to paramedic or emergency staff arrival or they received LDM as a second or later analgesic.

Across all countries, patients' mean (SD) age was 46.7 (20.2) years and ranged from 18 to 104 years (Table 1). Patients from Germany tended to be younger (mean [SD] 35.1 [10.7] years) while those in Spain were older (56.5 [18.1]). Across all countries, more male than female patients were enrolled ( $60 \%$ vs $40 \%$ ), consistent with data from the individual countries except for Belgium (50\% vs $50 \%$ ). Demographic characteristics of the patients enrolled in
Europe and Australia were broadly comparable, except for fewer Caucasian patients in the Australian cohort (94\% vs $78 \%$ ). Within the Australian cohort, there were no significant differences in demographic characteristics in the unmatched LDM vs non-LDM ( $\mathrm{n}=128$ vs $\mathrm{n}=118 ; P>0.05$ ) or PSM LDM and non-LDM ( $\mathrm{n}=85$ vs $\mathrm{n}=85 ; P>0.05$ ) groups.

\section{Injury and pain characteristics}

Broadly similar injury types were reported in Europe and Australia, with fractures being the most common injury type (38\% and $35 \%$, respectively [Table 2]). Fractures were most frequently reported in Belgium (50\%) and less common in the German cohort (21\%) where compressions were the most common injury (28\%). Sprains/strains were reported in $20 \%$ of the patients from both Europe and Australia while there was a higher proportion of dislocations ( $8 \%$ vs $17 \%)$ and lacerations ( $7 \%$ vs $17 \%)$ and fewer contusions ( $22 \%$ vs $10 \%)$ and compressions $(10 \%$ vs $2 \%)$ occurring in the latter (Table 2). While injury severity varied between individual countries, across Europe (64\%) and Australia (62\%) most injuries were rated as "moderate" (Table 2). NRS ( $\mathrm{n}=678$ ) was used more frequently that VAS $(\mathrm{n}=178)$ to assess patient-reported pain (Table 2). Moderate pain was the most common pain severity category reported across all countries ( $73 \%$ of the cases) with severe pain being reported in the remaining $27 \%$ of the cases.

There were some differences in injury type and pain characteristics between the unmatched LDM and nonLDM subgroups of Australian patients, including frequencies of fractures ( $43 \%$ vs $27 \%, P=0.0094$ ), contusions ( $5 \%$ vs $13 \%, P=0.0467)$ and compression injuries ( 0 vs $4 \%$, $P=0.0186$ ). Also, while moderate injury severity was more frequent in the LDM versus non-LDM subgroup (72\% vs $51 \%, P=0.0022)$, most patients reported moderate pain in both groups ( $74 \%$ vs $72 \%, P>0.05$ ). In the PSM LDM and non-LDM groups (both, $\mathrm{n}=85$ ) injury and pain characteristics were similar $(P>0.05$ all comparisons), with fractures $(35 \%$ vs $35 \%)$ and sprain/strain (19\% vs $21 \%)$ being the most frequent injury types. Injury severity was most commonly rated as moderate ( $58 \%$ vs $58 \%$ ), and most patients reporting moderate pain severity ( $74 \%$ vs $74 \%$ ) in the PSM, LDM and non-LDM groups.

\section{Pain relief treatment timelines}

The mean (SD) time from emergency call to arrival of the paramedic was 24.4 (18.9) mins across all countries, ranging from 13.1 mins in Belgium and Germany to 34.1 mins in Spain (Table 3). The mean (SD) time from 


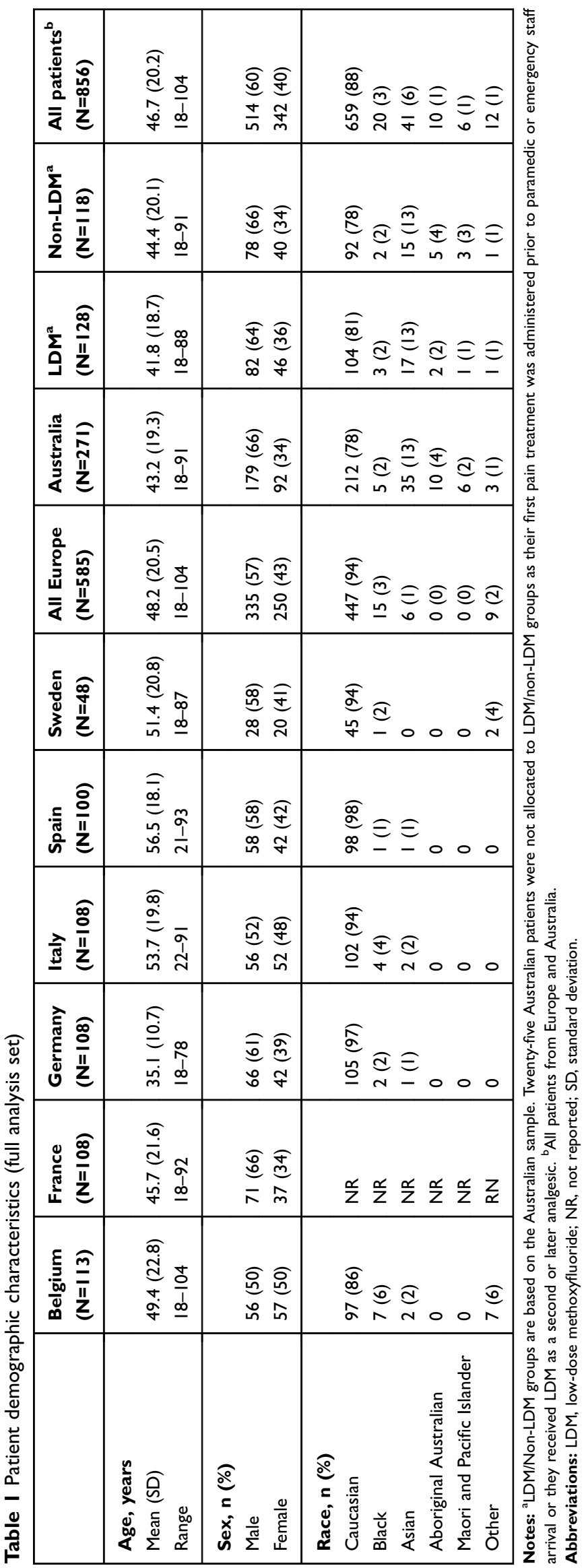




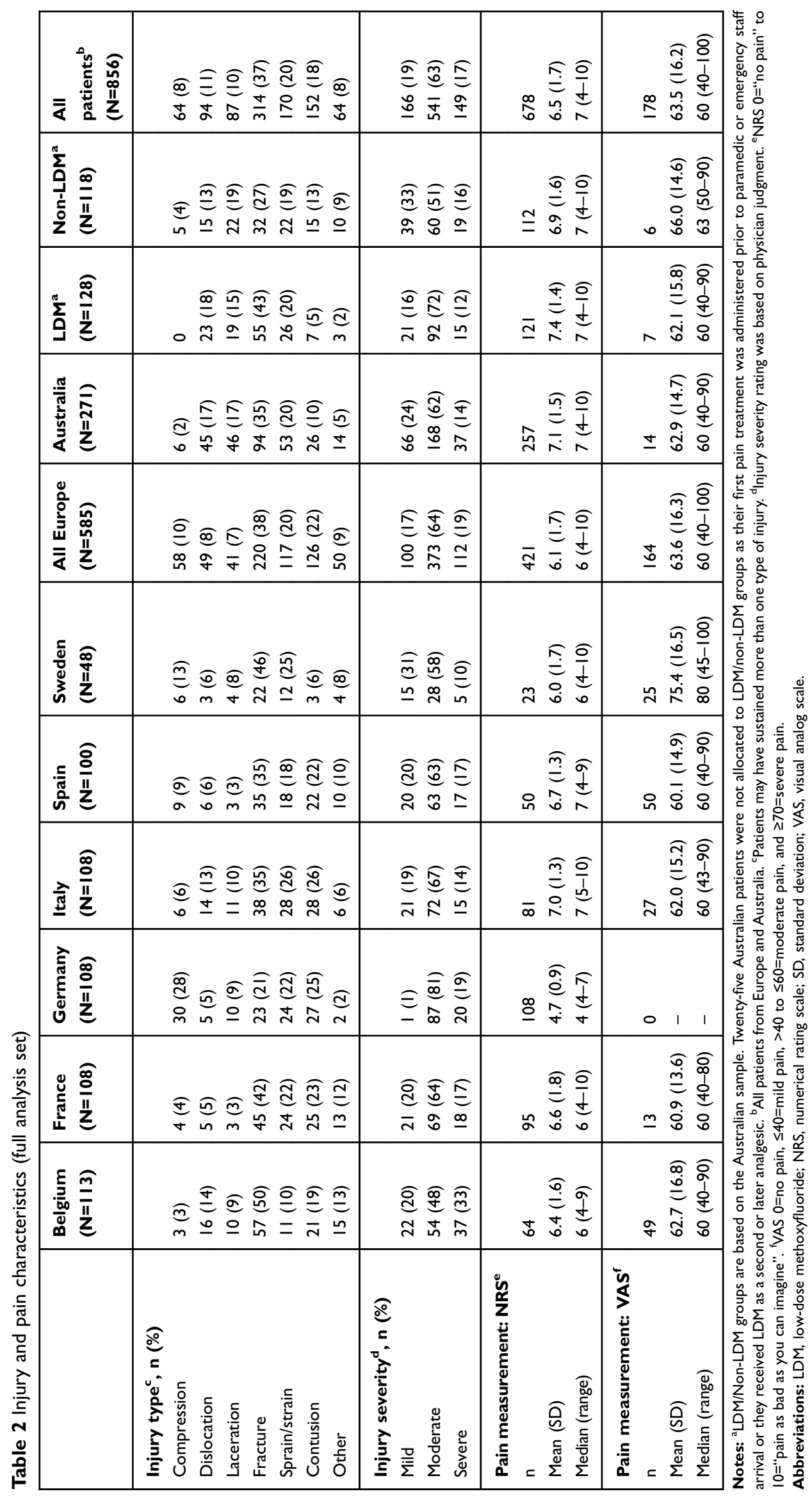




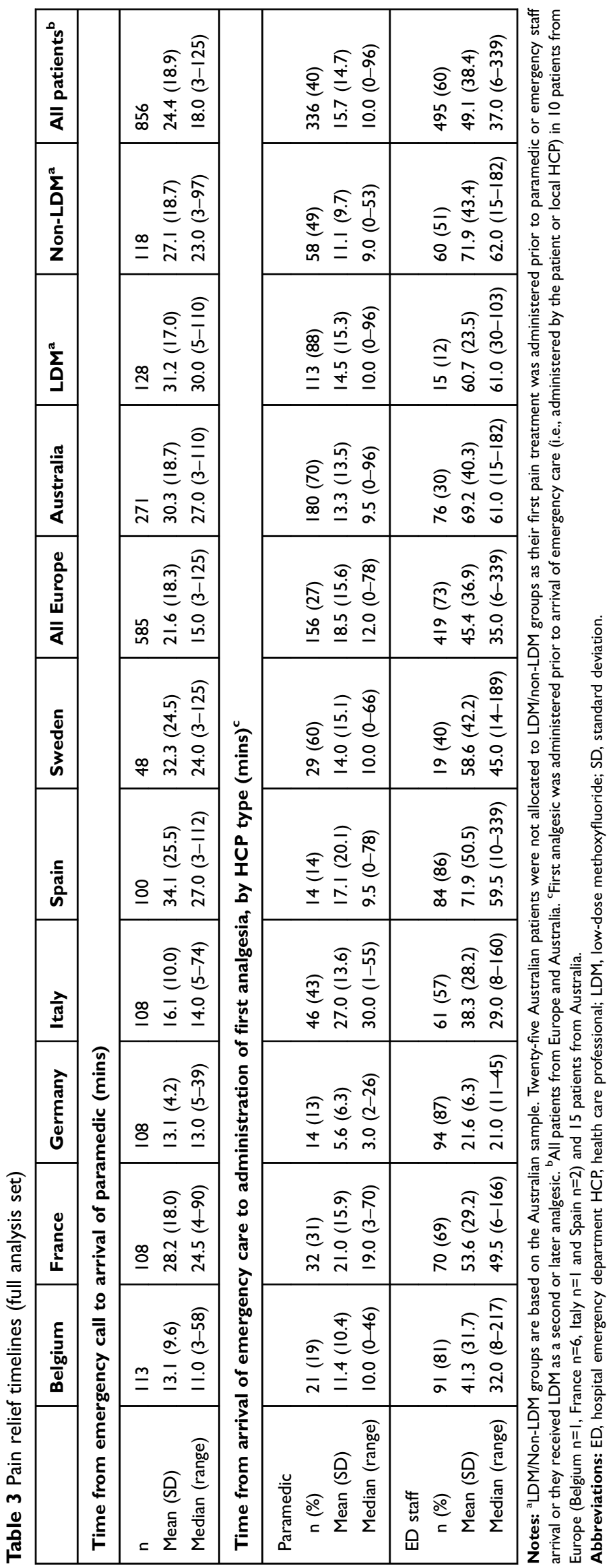


paramedic arrival to arrival at the ED was 25.9 (18.0) mins across all countries and ranged from 13.8 (5.0) mins in Germany to 31.8 (21.3) mins in France.

For the primary endpoint, time to first analgesic administration following arrival of paramedic or emergency assistance was 35.6 (35.1) mins across all countries and ranged from 19.5 (8.3) mins in Germany to 64.1 (51.0) mins in Spain (Figure 1). The mean (SD) time to first pain relief treatment was greater across the six European countries versus Australia (38.1 [34.7] vs 29.9 [35.5] mins, $P=0.0017$ [Figure 1]).

First analgesic was administered after arrival of emergency care by a paramedic or hospital ED staff member in most cases $(n=831$ [97\%]). Across all countries, for these patients, the mean (SD) time to first analgesic was shorter when the treatment was administered by paramedics versus hospital ED staff (15.7 [14.7] vs 49.1 [38.4] mins [Table 3]). In 5 of the 6 European countries combined, the first analgesic was provided by a paramedic in only $24 \%$ of the patients on average and was rarely administered in the ambulance, prior to arrival at the hospital ED $(10 \%)$. Sweden was the only European country in which the first analgesic was administered by paramedics $(60 \%)$ and provided in an ambulance (54\%) for most patients, as was also observed in Australia (70\% and 62\%, respectively). Subgroup analysis of the Australian cohort revealed a high proportion of patients received LDM during paramedic care $(88 \%)$ and while in an ambulance (80\%) compared with patients who received other analgesics (non-LDM: 49\% and 45\%, respectively). Also, the mean (SD) time to first pain relief treatment after arrival of emergency care (primary endpoint) was significantly lower in the LDM versus non-LDM group (PSM subgroups: mean [SD] 21.7 [24.2] vs 39.1 [43.0] mins, $P=0.0013$ : Figure 1). Similar findings were observed in the full analysis set (19.9 [22.1] vs 42.0 [43.9] mins, $P<0.0001)$ and in the non-parametric sensitivity analysis (median 11.0 vs 25.5 mins, $P<0.0001$ ).

There was no evidence for a relationship between time to pain treatment and time to discharge from hospital (correlations in all countries were $<0.3$ ). Unadjusted $(P=0.70)$ and adjusted $(P=0.86)$ linear regression models incorporating data from all countries also indicated no evidence of a relationship between time to pain treatment and time to hospital discharge.

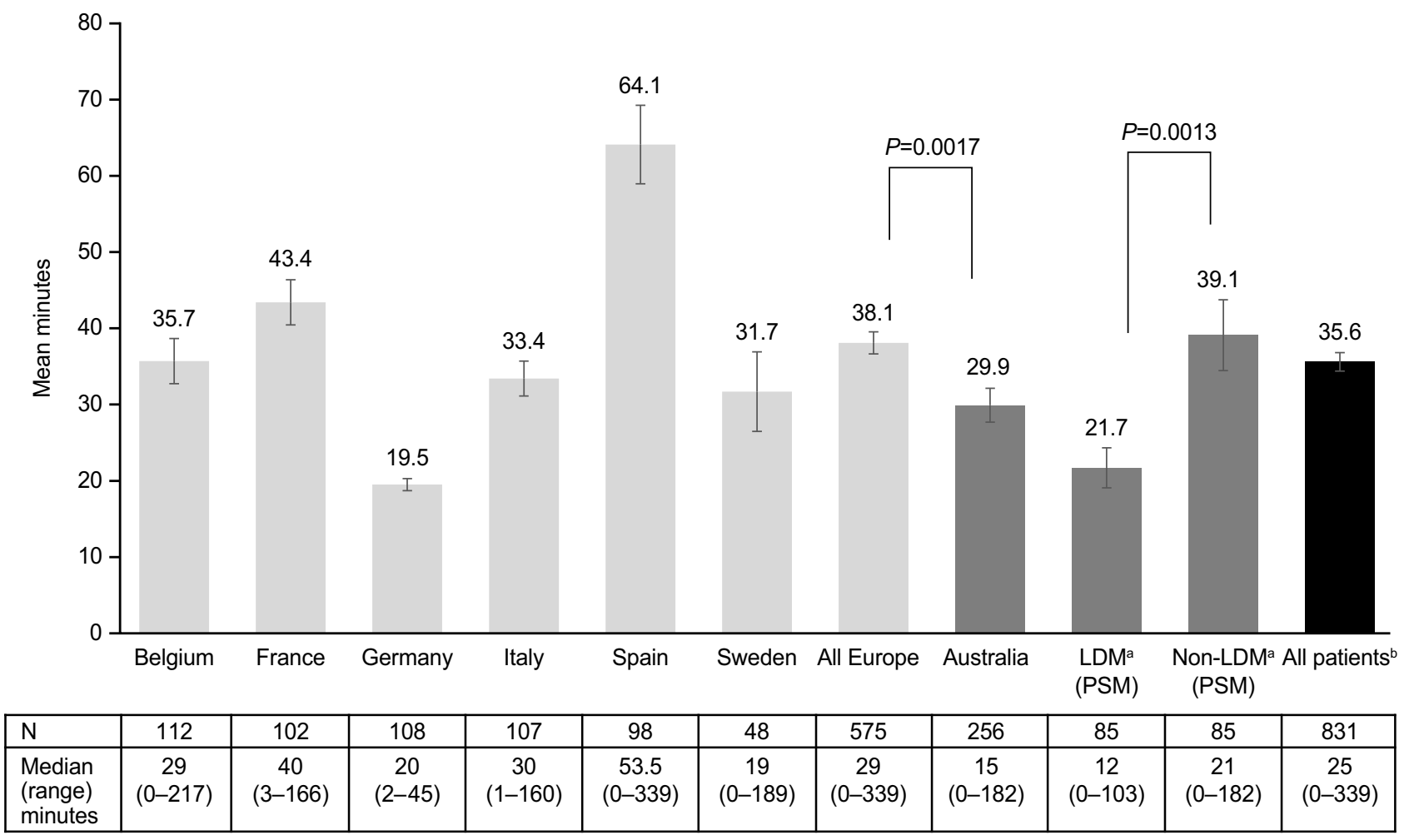

Figure I Time to first analgesic administration following arrival of paramedic or emergency assistance (full analysis set).

Notes: ${ }^{a}$ LDM/Non-LDM groups are based on the Australian sample. Twenty-five Australian patients were not allocated to LDM/non-LDM groups as their first pain treatment was administered prior to paramedic or emergency staff arrival or they received LDM as a second or later analgesic. ${ }^{b}$ All patients from Europe and Australia. Error bars \pm SEM.

Abbreviations: LDM, low-dose methoxyflurane; PSM, propensity score matched; SD, standard deviation. 


\section{Pain relief treatments}

A wide range of analgesics were administered as first pain relief treatment across Europe, with IV paracetamol $(\mathrm{n}=129$, $22 \%)$, IV NSAID $(n=114,19 \%)$, oral paracetamol $(n=98$, $17 \%)$ and IV opioid $(n=77,13 \%)$ being most frequently used in this setting (Figure 2). While LDM was rarely used in Europe ( $n=12,2 \%)$, it was the most frequent analgesic first administered to patients in Australia ( $n=129,48 \%$ ). IV opioid $(n=38,14 \%)$ and oral paracetamol $(n=25,9 \%)$ were the second- and third-most frequently administered first analgesics to patients in Australia, respectively (Figure 2).

Overall, $43 \%$ of the patients received subsequent pain treatment. The most frequently used subsequent pain medications in Europe were IV opioids $(n=63,11 \%)$, IV NSAIDs $(\mathrm{n}=55,9 \%)$ and IV paracetamol $(\mathrm{n}=51,9 \%)$. In Australia, there were $11(4.1 \%)$ instances where LDM was given as the subsequent analgesic medication, while strong oral opioid $(\mathrm{n}=50,18 \%)$, IV opioids $(\mathrm{n}=26,10 \%)$ and oral NSAIDs $(n=25,9 \%)$ were administered most frequently (Figure 3$)$.

\section{Discussion}

While effective pain management is an important part of emergency care, oligoanalgesia is frequently reported and can have a substantial impact on patients' physical and emotional wellbeing. ${ }^{2,6,11}$ Understanding pain treatments, timelines and outcomes in emergency care may provide insight into potential approaches to reduce the burden of trauma-related pain. To our knowledge, this is the first study to investigate the time to pain relief treatment experienced by patients with acute musculoskeletal injuries and moderate-to-severe pain who received emergency care by paramedics and/or hospital ED HCPs across 6 European countries and Australia. While LDM was approved in Europe for conscious adults with traumarelated pain in 2015, it was not widely available at the time of this analysis. Consequently, data from patients treated in emergency settings in Australia, where LDM is used extensive, were included in this analysis.

This analysis of real-world data from medical charts of 856 patients revealed substantial variation in the time to administration of first pain relief treatment following arrival of paramedic or ED HCP in Europe (mean [SD] 19.5 [8.3] mins in Germany to 64.1 [51.0] mins in Spain). Also, across Europe, patients experienced a longer mean [SD] time to administration of first pain relief treatment compared with Australia (38.1 [34.7] vs 29.9 [35.5] mins;

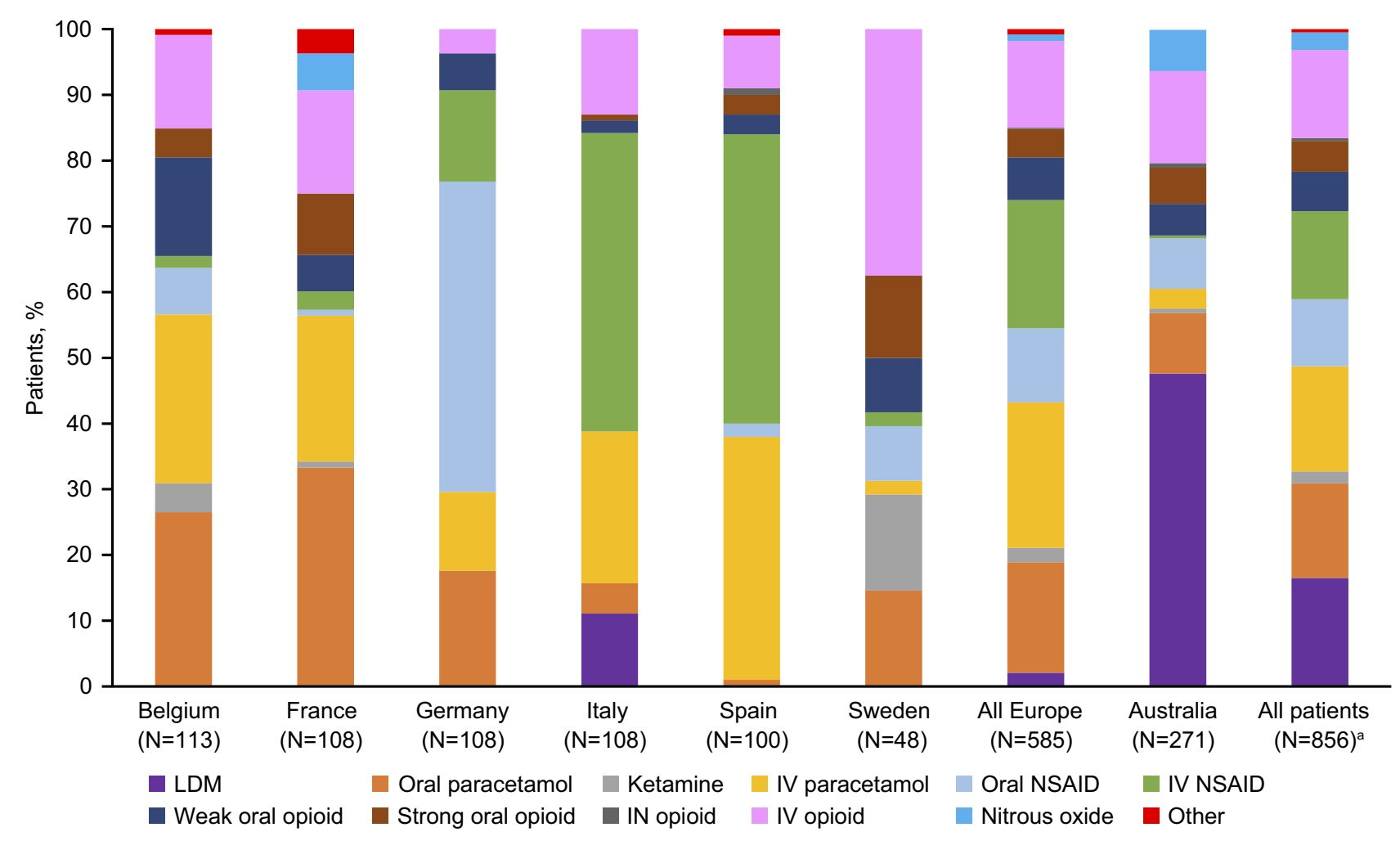

Figure 2 Initial analgesic administered (full analysis set).

Notes: ${ }^{\mathrm{A}}$ All patients from Europe and Australia.

Abbreviations: IN, intranasal; IV, intravenous; LDM, low-dose methoxyflurane; NSAID, non-steroidal anti-inflammatory. 


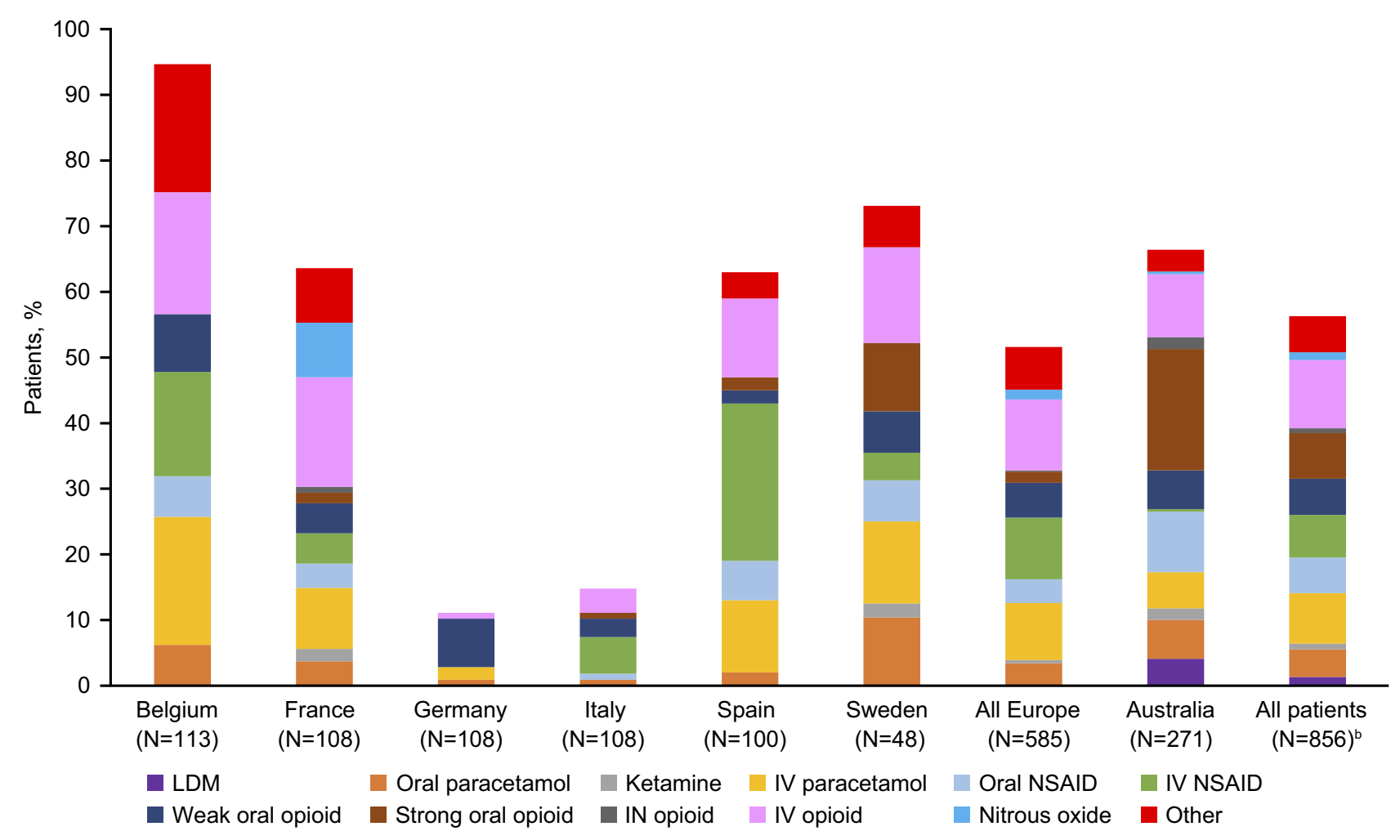

Figure 3 Subsequent analgesic administered (full analysis set) ${ }^{\mathrm{a}}$.

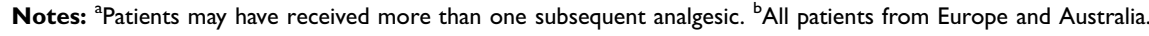

Abbreviations: IN, intranasal; IV, intravenous; LDM, low-dose methoxyflurane; NSAID, non-steroidal anti-inflammatory.

$P=0.0017)$. While local guidelines and treatment practices may account for some of this variation, these differences between countries require further investigation to understand how analgesic timelines can be optimized. Our data suggest wider use of LDM as the first analgesic in Australia compared with Europe (48\% vs $2 \%$ ) likely contributed to the timelier administration of pain treatment in Australia. For example, individuals in Australia received LDM much more quickly than other analgesics following arrival of emergency care (PSM subset: 21.7 [24.2] vs 39.1 [43.0] mins; $P=0.0013)$. Indeed, patients across Europe and all those in Australia who were not treated with LDM received their first analgesia in a similar time frame (38.1 [34.7] and 42 [43.9] mins, respectively).

An important observation from this real-world study was the substantially shorter time to pain treatment when analgesics were administered by paramedics compared with hospital ED HCPs (15.7 [14.7] vs 49.1 [38.4] mins following arrival of emergency care). While this may be unsurprising, given that paramedics are often the first HCP to attend injured patients, it is noteworthy that in 5 of the 6 European countries combined, the first analgesic treatment was rarely provided by paramedics $(24 \%)$ or administered in an ambulance (10\%). This observation suggests that increased utilization of analgesics by paramedics may enable more patients with acute, musculoskeletal injuries to receive analgesia promptly. Some of the variation in time to first analgesia may be attributed to regulatory barriers which prevent ambulance personnel from administering opioid analgesics in some countries. ${ }^{13}$ Furthermore, some paramedics and patients may express a preference for nonIV pain relief treatments. Consequently, it is feasible that greater utilization of non-opioid, needle-free analgesics by paramedics which can provide effective relief of moderateto-severe pain may result in a faster time to first pain relief treatment. This was evidenced by the use of LDM in Australia in the present study and is supported by a recent systematic review which concluded that while both LDM and $\mathrm{N}_{2} \mathrm{O}$ provide rapid analgesia in trauma settings, ease of administration and portability of LDM may be of significant value across a range of emergency care settings. ${ }^{19}$

Absence of a relationship between time to pain relief treatment and time to discharge from hospital demonstrated in this study is perhaps unsurprising, given there are many clinical considerations which influence time to hospital discharge that are unrelated to the timing of first pain treatment. 
These may include hospital occupancy, availability of medical staff, other treatment required for the underlying injuries, as well as variations in hospital protocols regarding monitoring time following strong pain relief treatments.

Given the geographic coverage provided by the 856 patient charts across seven countries from a large number of hospitals ( $n=145)$ and physicians ( $n=189)$, this study likely provides a robust picture of analgesic timelines for patients with acute musculoskeletal injuries in real-world practice compared with clinical studies that would typically involve a limited number of centers. Single-center observational studies reporting waiting times in excess of $40 \mathrm{mins}$ in European hospital ED for patients with moderate-to-severe pain support these findings. ${ }^{9,20}$ Nevertheless, this retrospective study was associated with some limitations. In order to study time to pain relief in a relatively homogenous population, this analysis focused on adults with moderate-to-severe pain associated with acute musculoskeletal injuries. While it is anticipated that these findings may be relevant for patients with moderate-to-severe resulting from other injuries or conditions, additional research in a broader emergency trauma population is warranted. Furthermore, while these data indicate that utilization of LDM in Europe may facilitate the timely relief of moderate-to-severe pain in emergency care to lessen patient suffering, further studies are needed to monitor the impact of LDM as a pain relief treatment option in Europe following its relatively recent approval.

In summary, this real-world observational study indicated an unmet need for timely administration of pain relief in patients with moderate-to-severe pain following musculoskeletal injury in Europe. Data from comparable patients receiving emergency care in Australia indicate that utilization of inhaled analgesics such as LDM by European paramedics could result in more timely pain relief for appropriately selected patients.

\section{Data availability}

Proposals for data access should be sent to the corresponding author.

\section{Acknowledgment}

The authors would like to thank Louise Hegarty for help with developing charts and figures. Medical writing support was provided by Siân Marshall of SIANTIFIX Ltd., Cambridgeshire, UK. Mundipharma International Ltd. funded this research. Mundibiopharma Ltd. funded medical writing support for the development of this manuscript.

\section{Disclosure}

Andrew D Xia was an employee at Mundipharma International Ltd. at the time this study was conducted and is now a consultant to Mundibiopharma Ltd. Sara L Dickerson is an employee of Mundibiopharma Ltd. Mika Nokela, Sam Colman and Agota Szende are employees of Covance Market Access Inc., providing research contracted by Mundipharma. The authors report no other conflicts of interest in this work.

\section{References}

1. Kisser R, Walters A, Rogmans W, Turner S, Lyons RA. Injuries in the European Union 2013-2015 (supplementary report); 2017. Available from: http://www.eurosafe.eu.com/uploads/inline-files/IDB\%2020132015 suppl\%20to\%206th\%20edition $\% 20$ Injuries $\% 20$ in $\% 20$ the $\%$ 20EU.pdf. Accessed April 18, 2019.

2. Sinatra R. Causes and consequences of inadequate management of acute pain. Pain Med. 2010;11(12):1859-1871. doi:10.1111/j.15264637.2010.00983.x

3. Glowacki D. Effective pain management and improvements in patients' outcomes and satisfaction. Crit Care Nurse. 2015;35(3):3341. doi: $10.4037 / \mathrm{ccn} 2015440$

4. Royal College of Emergency Medicine. Best practice guideline: management of pain in adults. Available from: https:/www.rcem.ac. uk/docs/College\%20Guidelines/5w.\%20Management $\% 20$ of\%20Pain \%20in\%20Adults\%20(Revised\%20December\%202014).pdf. Accessed April 18, 2019.

5. Tamches E, Buclin T, Hugli O, et al. Acute pain in adults admitted to the emergency room: development and implementation of abbreviated guidelines. Swiss Med Wkly. 2007;137(15-16):223-227. doi:2007/15/smw11663

6. Mura P, Serra E, Marinangeli F, et al. Prospective study on prevalence, intensity, type, and therapy of acute pain in a second-level urban emergency department. J Pain Res. 2017;10:2781-2788. doi:10.2147/JPR.S137992

7. Dale J, Bjornsen LP. Assessment of pain in a Norwegian Emergency Department. Scand J Trauma Resusc Emerg Med. 2015;23:86. doi:10.1186/s13049-015-0166-3

8. Boccard E, Adnet F, Gueugniaud PY, Filipovics A, Ricard-Hibon A. Pain management in adult patients in emergency care units in France in 2010 [French, English]. Ann Francauses de Med d'Urgence. 2011;1(5):312-319. doi:10.1007/s13341-011-0094-4

9. Karwowski-Soulie F, Lessenot-Tcherny S, Lamarche-Vadel A, et al. Pain in an emergency department: an audit. Eur J Emerg Med. 2006;13(4):218-224. doi:10.1097/01.mej.0000217975.31342.13

10. Oberholzer N, Kaserer A, Albrecht R, et al. Factors influencing quality of pain management in a physician staffed helicopter emergency medical service. Anesth Analg. 2017;125(1):200-209. doi:10.1213/ ANE.0000000000002016

11. Albrecht E, Taffe P, Yersin B, Schoettker P, Decosterd I, Hugli O. Undertreatment of acute pain (oligoanalgesia) and medical practice variation in prehospital analgesia of adult trauma patients: a $10 \mathrm{yr}$ retrospective study. Br J Anaesth. 2013;110(1):96-106. doi:10.1093/bja/aes355

12. Dissmann PD, Maignan M, Cloves PD, Gutierrez Parres B, Dickerson S, Eberhardt A. A review of the burden of trauma pain in emergency settings in Europe. Pain Ther. 2018;7(2):179-192. doi:10.1007/s40122-018-0101-1

13. Walsh B, Cone DC, Meyer EM, Larkin GL. Paramedic attitudes regarding prehospital analgesia. Prehosp Emerg Care. 2013;17 (1):78-87. doi:10.3109/10903127.2012.717167 
14. Porter KM, Dayan AD, Dickerson S, Middleton PM. The role of inhaled methoxyflurane in acute pain management. Open Access Emerg Med. 2018;10:149-164. doi:10.2147/OAEM.S181222

15. Craigavon, UK:Galen Limited. Penthrox $3 \mathrm{~mL}$ inhalation vapour liquid: summary of product characteristics. 2016; Available from: http://www.medicines.org.uk/emc/medicine/31391. Accessed April 18, 2019.

16. Grindlay J, Babl FE. Review article: efficacy and safety of methoxyflurane analgesia in the emergency department and prehospital setting. Emerg Med Australas. 2009;21(1):4-11. doi:10.1111/ j.1742-6723.2009.01153.x

17. Bendall JC, Simpson PM, Middleton PM. Prehospital analgesia in New South Wales, Australia. Prehosp Disaster Med. 2012;26(6):422426. doi:10.1017/S1049023X12000180
18. Coffey F, Wright J, Hartshorn S, et al. STOP!: a randomised, doubleblind, placebo-controlled study of the efficacy and safety of methoxyflurane for the treatment of acute pain. Emerg Med J. 2014;31 (8):613-618. doi:10.1136/emermed-2013-202909

19. Porter KM, Siddiqui MK, Sharma I, Dickerson S, Eberhardt A. Management of trauma pain in the emergency setting: low-dose methoxyflurane or nitrous oxide? A systematic review and indirect treatment comparison. J Pain Res. 2018;11:11-21. doi:10.2147/JPR.S150600

20. Kone V, Lecomte F, Randriamanana D, Pourriat JL, Claessens YE, Vidal-Trecan G. Impact of a pilot team on patients' pain reduction and satisfaction in an emergency department: A before-and-after observational study. Rev Epidemiol Sante Publique. 2016;64(2):5966. doi:10.1016/j.respe.2015.11.010

\section{Publish your work in this journal}

The Open Access Emergency Medicine is an international, peerreviewed, open access journal publishing original research, reports, editorials, reviews and commentaries on all aspects of emergency medicine. The manuscript management system is completely online and includes a very quick and fair peer-review system, which is al easy to use. Visit http://www.dovepress.com/testimonials.php to read real quotes from published authors. 